\title{
GTOBAS: fitting continuum functions with Gaussian-type orbitals
}

\author{
Alexandre Faure, Jimena D. Gorfinkiel, Lesley A. Morgan, \\ Jonathan Tennyson $^{1}$ \\ Department of Physics and Astronomy, University College London, Gower Street, \\ London WC1E $6 B T, U K$
}

GTOBAS is a program for fitting Gaussian-type orbitals (GTOs) to Bessel and Coulomb functions over a finite range. The exponents of the GTOs are optimised using the method of Nestmann and Peyerimhoff (J. Phys. B, 23 (1990) L773). The appended module NUMCBAS provides the numerical Bessel and Coulomb functions required as input for the program. The use of GTO continuum basis sets is particularly important in electronmolecule scattering calculations when polyatomic targets are involved. Sample results for such calculations are also discussed.

Key words:

Gaussian basis sets, Bessel and Coulomb functions, R-matrix calculations

PACS: 02.60.Ed, 34.80.-i, 52.20.Fs

\section{PROGRAMS SUMMARY}

\section{GTOBAS}

Title of program: GTOBAS

Catalogue identifier: (supplied by Elsevier)

Program obtainable from: CPC Program Library, Queen's University of Belfast, N. Ireland

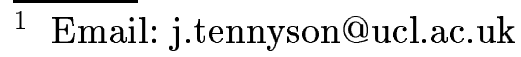

Preprint submitted to Elsevier Preprint $\quad 7$ September 2001 
Computer for which the program is designed and others on which it has been tested: Compaq Alpha-DEC, IBM RS/6000

Operating systems or monitors under which the program has been tested: Digital UNIX V5.0, IBM AIX 4.3.2.0

Programming language used: Fortran 90

Memory required to execute with typical data: less than 0.25 Mwords

No. of bits in a word: 32

No. of processors used: 1

Has the code been vectorised?: No

No. of bytes in distributed program, including test data,etc.: 29117

Distribution format: ASCII

Keywords: Gaussian basis sets, Bessel and Coulomb functions, R-matrix calculations

Nature of physical problem

Optimising GTO basis sets to represent continuum functions.

Method of solution

Numerical continuum functions are read from an external file and the GTOs set is optimised using the method proposed by Nestmann and Peyerimhoff [1]. 
Restrictions on the complexity of the problem

The limitation for obtaining satisfactory fits is linked to the intrinsic difficulty of representing a large number of nodes with nodeless Gaussian functions expanded about a single centre.

Typical running time

1 to 10 minutes (depending on the number of needed GTOs) plus the time taken by the subroutine used to generate the numerical functions.

Unusual features of the program

The program makes use of subroutines from Numerical Recipes [2]. We also append a module, NUMCBAS, for generating Bessel and Coulomb functions (see below).

\section{NUMCBAS}

Title of program: NUMCBAS

Catalogue identifier: (supplied by Elsevier)

Program obtainable from: CPC Program Library, Queen's University of Belfast, N. Ireland

Computer for which the program is designed and others on which it has been tested: Compaq Alpha-DEC, IBM RS/6000

Operating systems or monitors under which the program has been tested: Digital UNIX V5.0, IBM AIX 4.3.2.0

Programming language used: Fortran 90 
Memory required to execute with typical data: less than 0.1 Mwords

No. of bits in a word: 32

No. of processors used: 1

Has the code been vectorised?: No

No. of bytes in distributed program, including test data,etc.: 24133

Distribution format: ASCII

Keywords: Bessel and Coulomb functions

Nature of physical problem

Evaluation/calculation of Bessel and Coulomb functions. Provides input for GTOBAS.

Method of solution

Numerical integration of the Schrödinger equation from both boundaries and matching using de Vogelaere's algorithm.

Restrictions on the complexity of the problem

The program could in principle solve a model scattering problem with any kind of potential, although in its present form, only Coulomb potentials can be input.

Typical running time

Typically, 0.03s.

References 
[1] B. M. Nestmann and S. Peyerimhoff, J. Phys. B: At. Mol. Opt. Phys. 23 (1990) L773.

[2] W. H. Press, B. P. Flannery, S. A. Teukolsky and W. T. Vetterling, Numerical Recipes in Fortran (Cambridge University Press, 1995). 


\section{LONG WRITE-UP}

\section{Introduction}

Standard quantum chemistry packages routinely use Gaussian-type orbitals (GTOs) to describe the electronic structure of nonlinear molecules. Although Slater-type orbitals (STOs) have the proper "cusp" near nuclei and the correct asymptotic behaviour, their use is almost totally confined to atomic and linear molecule calculations because the multicenter integrals that arise in nonlinear calculations cannot be performed efficiently (see e.g. [1]). In contrast, such integrals can routinely be evaluated (in closed form) when GTOs are used. This fundamental advantage has lead to the dominance of GTOs in molecular electronic structure calculations.

A huge number of GTO basis sets have been made available for bound-state calculations [2]. On the other hand, few basis set generation procedures exist for the representation of Rydberg and continuum orbitals [3]. These functions cover a large spatial domain and their radial part is characterised by a great number of nodes. In the context of electron-molecule scattering calculations, the use of numerical functions to represent the continuum has proved very successful for both atomic and linear targets [4]. For nonlinear molecules, however, there are at present no adequate numerical procedures available. An alternative approach, explored originally in the context of R-matrix calculations, involves the use of GTOs to represent both the continuum and target electrons. Nestmann \& Peyerimhoff [5] developed a method to fit GTO basis sets to Bessel functions within the finite region of an R-matrix sphere. In spite of the intrinsic difficulty of representing a large number of nodes with (nodeless) Gaussian functions expanded about a single centre, this method was found to give excellent results for Bessel functions with eigenenergies up to $16 \mathrm{eV}$. The method has been used to obtain a number of GTO continuum basis sets, all for neutral targets and to be used in calculations with an R-matrix radius of $R_{m a t}=10 \mathrm{a}_{0}$ [5-7]. A general discussion on approximating functions with non-orthogonal basis sets can be found in [8].

The aim of this work is to provide a program which is able to construct adequate GTO continuum basis sets for representing both Bessel and Coulomb functions using the procedure described by Nestmann \& Peyerimhoff and to briefly discuss the behaviour of these basis sets in actual calculations. We also append the module NUMCBAS, based on a program by Salvini [9] and which is a cut-down version of the module NUMBAS used in the UK molecular R-matrix codes [10] to generate the numerical continuum orbitals. The paper is organised as follows: Section 2 summarises the method used to optimise the Gaussian exponents while Section 3 reports and discusses sample results. The program organisation and input data description are given in Section 4. Test data is given in Section 5. 


\section{Method}

We summarise here the procedure used in GTOBAS to optimise the Gaussian exponents. A brief description of the module NUMCBAS, used to generate numerical Bessel and Coulomb functions, is also given. It should be noted that any other usersupplied program can be used to produce these functions, or indeed other functions that one may wish to fit.

\subsection{Generation of numerical continuum orbitals}

The module NUMCBAS defines an adaptive grid of radial coordinates $r_{k}$ to generate numerical continuum orbitals $u_{h l}\left(r_{k}\right)$ by solving the model, single channel scattering equation:

$$
\left[\frac{d^{2}}{d r_{k}^{2}}-\frac{l(l+1)}{r_{k}^{2}}+2 V_{0}+k_{h}^{2}\right] u_{h l}\left(r_{k}\right)=0
$$

where $l$ is the angular momentum quantum number, $k_{h}^{2}$ are the eigenenergies and $V_{0}$ is a model potential, both in Rydbergs. This equation is solved subject to the fixed boundary conditions:

$$
u_{h l}(0)=0 \text { for } l \neq 0, \quad \frac{1}{u_{h l}\left(r_{k}\right)}\left[\frac{d u_{h l}\left(r_{k}\right)}{d r_{k}}\right]_{r_{k}=R_{\mathrm{lim}}}=0
$$

where $R_{\text {lim }}$ is the boundary radius in Bohrs $\left(r_{k} \leq R_{\text {lim }}\right)$. For the special case $l=0$ the value of $u_{h l}(0)$ is obtained by a two point Lagrange interpolation. Only those eigenfunctions whose energy $k_{h}^{2}$ is smaller than the parameter $E_{\text {up }}$ (typically a few Rydbergs) are evaluated. NUMCBAS finds solutions to Eq. 1 by integrating functions from both boundaries and matching them using de Vogelaere's algorithm $[11,12]$. It has been found that, in practice, $V_{0}$ in Eq. 1 can be replaced by $Z / r_{k}$ where $Z$ is the effective charge of the target. In this case, the eigensolutions become numerical representations of spherical Bessel $(Z=0)$ or Coulomb functions over a finite range.

\subsection{Optimisation procedure}

The procedure used to obtain the Gaussian exponents was originally proposed by Nestmann \& Peyerimhoff [5]. The main idea of this scheme is to fit the continuum functions $u_{h l}\left(r_{k}\right)$ by a set of Gaussian functions with exponents $\alpha_{i}$. This is performed 
by minimising the function:

$$
F_{l, N}=\sum_{h=1}^{N} \frac{\sum_{k}\left[\sum_{i=1}^{n} c_{h i} r_{k}^{l} \exp \left(-\alpha_{i} r_{k}^{2}\right)-u_{h l}\left(r_{k}\right)\right]^{2}}{\sum_{k}\left[u_{h l}\left(r_{k}\right)\right]^{2}}+D\left(\alpha_{1}, \ldots, \alpha_{n}\right)
$$

where $N$ is the number of continuum functions and $n$ is the number of Gaussian exponents. The minimum of $F_{l, N}$ is obtained by Powell's method [13] (prototype of multidimensional direction-set methods) using $\ln \left(\alpha_{i}\right)$ as variational parameters. The coefficients $c_{h i}$ are determined by a least-squares fit.

The term $D\left(\alpha_{1}, \ldots, \alpha_{n}\right)$ :

$$
D\left(\alpha_{1}, \ldots, \alpha_{n}\right)=\sum_{i=2}^{n} \sum_{j=1}^{i-1} \exp \left(-g \times\left|\frac{\alpha_{i}}{\alpha_{j}}-\frac{\alpha_{j}}{\alpha_{i}}\right|\right),
$$

is added to avoid the convergence of two different $\alpha_{i}$ towards the same value and hence problems with linear dependence. In Eq. 4 the real number $g$ has been set to $R_{\text {lim }}$, the boundary radius defined in NUMCBAS (see Eq. 2).

It is important to note that the logarithmic boundary condition defined in Eq. 2 leads to an artificial constraint on the numerical continuum functions at $r_{k}=R_{\text {lim }}$. Employing the R-matrix technique, the continuum basis set must be able to describe both maxima and nodes at the boundary radius of the $\mathrm{R}$-matrix sphere, $R_{\text {mat }}$. As a consequence, $R_{\text {lim }}$ must be chosen larger than $R_{\text {mat }}$. In practice, we found for $R_{\text {mat }}$ in the range 10-13 a (the only values thoroughly tested) that $R_{\text {lim }}=R_{\text {mat }}+2 \mathrm{a}_{0}$ is the best choice for both Bessel and Coulomb functions (see details in Section 3).

\subsection{Initial selection of exponents}

The minimisation problem is highly non-linear and characterised by having many local minima. This makes the final fit sensitive to the starting point. In our procedure, the initial Gaussian exponents $\alpha_{i}$ can be read directly as input data. Alternatively, we implemented two different methods of selection. In the first one, the initial exponents are selected randomly within an appropriate range $([0.01,0.5]$ is the default). In the second, recommended method, a geometric series is used to generate the initial, even-tempered [14], set of exponents:

$$
\alpha_{i}=\beta \times \gamma^{i} ; \quad i=1,2,3, \ldots, n .
$$

In the present work, the default values are $\beta=0.016$ and $\gamma=1.39$ for both Bessel and Coulomb functions. These values were obtained by fitting and averaging (over the different $l$ values) typical final sets of exponents. The use of Eq. 5 as an initial guess was found to minimise the required CPU time. 


\section{Sample results and discussion}

In this section we present GTO basis sets optimised with GTOBAS for the representation of Bessel and Coulomb functions. These continuum basis sets have been employed in electron-molecule scattering calculations $[15,16]$ using the UK R-matrix polyatomic codes [10]. Bessel and Coulomb functions with values $l=0,1, \ldots, 4$ were generated with NUMCBAS. The main input parameters, namely $R_{\text {lim }}, E_{\text {up }}$ and the number $n$ of GTOs, have been determined by the needs of the molecular systems studied and by the limitations of the computer resources. The radial mesh was chosen to be finer at the shorter ranges. Sensible changes in the mesh have little influence on the final value of the Gaussian exponents (typically less than 5\%). The value of $R_{l i m}$ is fixed by the value of the R-matrix radius (see 2.2). The upper energy, $E_{\mathrm{up}}$, then determines the number of numerical functions to be fitted and therefore, the number of GTOs which must be bigger than or equal to the number of numerical functions. Note that there are no restrictions on the upper values of $R_{\text {lim }}$ and $E_{\text {up }}$ other than the required computational time (e.g. up to 70 minutes for $R_{\text {lim }}=20 \mathrm{a}_{0}, E_{\mathrm{up}}=7$ Ryd and 17 GTOs).

Fig. 1 and Fig. 2 show, respectively, $l=0$ Bessel and Coulomb functions and their corresponding fits. The region of best fit, $0<r_{k}<3$, is omitted from the figures for clarity. Although functions with $l=0$ are the hardest to fit, it can be seen that, in general, the agreement is very good. As expected, when the number of nodes increases, the quality of the fit decreases. Besides, as $r_{k}$ increases, the quality of the fit decreases too. A number of weighting procedures were tested to improve the fits at large $r_{k}$, without success. However, in R-matrix calculations, only the region $r_{k} \leq R_{\text {mat }}$ is considered; therefore, the quality of the fit for $r_{k}>R_{\text {mat }}$ is not relevant. The accuracy of the fit can be assessed by checking the final value of the minimisation function $F_{l, N}$ (see Eq. 3): for the fits presented in Figs. 1-2, $F_{l, N}$ was found to be smaller than $4 \times 10^{-3}$.

When using the R-matrix method, the set of continum functions has to be complete within the finite region of the R-matrix sphere $\left(0<r_{k}<R_{\text {mat }}\right)$. A basic difficulty is that this can cause problems of linear dependence with the functions representing the target [4]. If the boundary radius $R_{\text {lim }}$ defined in NUMCBAS is much larger than the R-matrix sphere radius $R_{\text {mat }}$, the continuum basis set can become overcomplete in the inner region defined by this sphere, leading to strong linear dependence problems. After several tests with $R_{\text {mat }}$ in the range 10-13 $\mathrm{a}_{0}$, we concluded that the best choice for the boundary radius (in terms of linear dependence) is $R_{\text {lim }}=$ $R_{\text {mat }}+2 a_{0}$, for both neutral and singly charged targets. Using a smaller $R_{\text {lim }}$ would produce a poor fit for $r_{k} \simeq R_{\mathrm{lim}}$. In their paper, Nestmann and Peyerimhoff suggested $R_{\text {lim }}=20 a_{0}$ for $R_{\text {mat }}=10 a_{0}$ but we found that using this value lead to severe linear dependence.

GTO basis sets for the representation of Bessel and Coulomb functions are given in Tables 1-3. Using a much larger number of GTOs than numerical functions can lead to some exponents being zero and/or more than one converged set. If the latter is the case, the quality of the different converged sets is very similar, at least in 
terms of its adequacy for scattering calculations. Finally, it should be noted that the fitting procedure given here also provides contraction coefficients for the GTO continuum basis. However, to maximise the flexibility of the basis in the R-matrix calculations, our practice, and that of Nestmann and co-workers [5-7], has been to leave these functions uncontracted.

\subsection{Neutral targets}

Table 1 compares our basis set for a neutral target and $R_{\text {mat }}=10 a_{0}$ with that obtained by Sarpal et al. [6] who also used the method of Nestmann and Peyerimhoff [5]. The differences between the two sets may arise from the use of a different boundary condition. Besides, Sarpal et al. use the same $E_{\text {up }}$ but do not specify their value of $R_{\mathrm{lim}}$. The most significant differences between the two sets are: $i$ ) for $l=0$, our basis set has 3 exponents that are bigger than their biggest one; ii) for the other $l$ values, the smallest exponents in our basis set are smaller than in their basis set.

In order to assess the validity of our basis set and how it compares with that of Sarpal et al., we used both sets to study the electron-impact electronic excitation of $\mathrm{H}_{2} \mathrm{O}$ [16] and $\mathrm{CF}_{2}$ [17]. Our basis set was found to give better eigenphases for both systems, as seen for the electron- $\mathrm{H}_{2} \mathrm{O}$ collision in Fig. 3, where we have plotted the eigenphase sum of symmetry ${ }^{2} \mathrm{~A}_{1}$ (in a variational calculation, a higher eigenphase indicates better results). The three plotted eigenphases are very similar in the energy region $0<\mathrm{E}<5 \mathrm{eV}$ but differences increase at energies above the first excitation threshold. Additionally, after performing several tests with different basis sets for both targets, we found that Sarpal et al.'s is more prone to linear dependence with the target basis set. This behaviour is, of course, target dependent.

Table 2 gives Gaussian exponents for the representation of Bessel functions generated with $R_{\lim }=15 a_{0}$. To adapt the size of the basis set to the computational limits imposed by the R-matrix code, we chose different $E_{\text {up }}$ for different $l$ values. In this way, we reduced the number of GTOs required for fitting the functions with higher $l$. The exponents, with exception of the first ones for $l=0$ and $l=1$, are smaller than those obtained with $R_{\mathrm{lim}}=12 a_{0}$, as expected. This basis set has been used for $\mathrm{H}_{2} \mathrm{O}$ calculations with an R-matrix radius of $13 a_{0}$ [16]. The resulting eigenphase sum and cross sections were found to be in very good agreement with those obtained for $R_{\text {mat }}=10 a_{0}$ using the basis set from Table 1 (see Fig. 3 ), thus proving the adequacy of this basis set. The increasing discrepancies at higher energies are probably due to the incompleteness of the basis set of Table 2 for $R_{\text {mat }}=13 a_{0}$.

\subsection{Ionic targets}

Gaussian exponents for singly charged targets are listed in Table 3. It can be noticed that for $l=0$ and $l=1$ some exponents are larger than unity. Such large values are 
consistent with the high amplitudes of $l=0,1$ Coulomb functions at short-range. Note that in this case, an even-tempered initial selection is not the most appropriate.

The basis set presented in Table 3 has been used to study the electron-impact rotational excitation of $\mathrm{HCO}^{+}[15]$ and $\mathrm{H}_{3}^{+}$[18]. In contrast to Bessel functions, there are no previous studies on the representation of Coulomb functions by GTOs. There is, however, a study on the dissociative recombination of $\mathrm{H}_{3}^{+}$by Orel and Kulander [19], where the scattering calculations are based on the complex Kohn variational method. In this approach, analytic Coulomb functions can be used. At the equilibrium geometry of the ion (for ${ }^{2} A_{1}$ symmetry), Orel and Kulander found that the two first resonance energies (widths) are 9.1 (0.64) and $10.3(0.18) \mathrm{eV}$. We computed these resonances using the R-matrix method and we obtained, respectively, $9.2(0.68)$ and $10.2(0.18) \mathrm{eV}$. This very good agreement with the results of Orel and Kulander indicates the reliability of our representation of the continuum. In order to further illustrate the influence of the continuum basis set on a scattering calculation, we have computed the eigenphase sum for the e- $\mathrm{H}_{3}^{+}$collision in ${ }^{2} \mathrm{~A}_{1}$ symmetry using our basis set (Table 3) and that of Sarpal et al. (1996), which was optimised to represent Bessel functions. The results, plotted in Fig. 4, show that our basis set gives better (higher) eigenphases, as expected. They also show that Sarpal et al.'s basis set gives somewhat oscillatory behaviour at lower energies (not plotted).

\section{Programs organisation and data input}

\subsection{Organisation of GTOBAS}

The subroutines in GTOBAS are mainly taken from Numerical Recipes [20]. Further comments are given within the code.

READNUM reads the numerical continuum functions produced by NUMCBAS. It can be user-adapted to read any other input continuum functions.

FUNCS computes a Gaussian-type function.

FUNCP computes the function $F_{l, N}$ (Eq. 3) to minimise.

SVDFIT performs a least-square fit by use of a singular value decomposition technique to obtain the coefficients $c_{h i}$ of the Gaussian expansion (see Section 2.2). It is taken from Numerical Recipes [20].

SVBKSB and SVDCMP are used by SVDFIT to perform the singular value decomposition $[20]$.

POWELL performs the minimisation of the function $F_{l, N}$ (Eq. 3). It is taken from Numerical Recipes [20]. 
LINMIN implements the one-dimensional line minimisation used by the POWELL subroutine [20].

MNBRAK is used by LINMIN to bracket a one-dimensional minimum [20].

\subsection{Organisation of NUMCBAS}

BASIS performs the calculation of the numerical basis.

SEARCH is used by BASIS to search for the eigensolutions to the differential equations (see Eq. 1).

BASFUN controls the numerical integration and normalises the resulting wavefunctions.

DEVGL is a de Vogelaere integration routine [11] used by BASFUN.

FINDER locates the eigenvalue with a given number of nodes.

WRHEAD writes the header of file lunumb which will contain the numerical basis in a form suitable for GTOBAS.

\subsection{Input data for GTOBAS}

Input data [with defaults in brackets] is read from standard input via a namelist /FIT/. The data type is indicated in the following way: variables starting with $(\mathrm{a}-\mathrm{h}, \mathrm{o}-\mathrm{z})$ are double precision and those starting with (i-n) are integers.

beta $\quad[1.6 \mathrm{D}-02]$

Even-tempered $\beta$ coefficient, see Eq. 5 (used if iguess=2).

expo Vector containing initial exponents. Only needed if iguess $=0$. Maximum size is 20 .

ftol [1.D-09]

Convergence parameter used in POWELL subroutine.

gamma $[1.39 \mathrm{D} 0]$

Even-tempered $\gamma$ coefficient, see Eq. 5 (used if iguess=2).

iguess $\quad[2]$

Determines whether initial exponents will be read as input data (0), generated randomly (1), or generated using an appropriate function (2) (see Section 2.3). 
iprint $\quad[0]$

Print flag for additional output. 1: all iteration data; 2: iteration data plus mesh.

iswmol3 [18]

Logical unit for the output of final exponents. The format is that of the Sweden-Molecule program $[21,22]$ used by the UK molecular R-matrix polyatomic code.

lunumb $[13]$

Logical unit for the input of numerical functions to be fitted.

luplot [17]

Logical unit for the output fitted functions. Numerical functions are also written to this file. The format allows plotting with xmgr.

noexp $[0]$

Number of exponents to be used. Must be bigger or equal to the number of numerical functions to be fitted. Only needed if iguess $=1$ or 2 .

nplot $[0]$

Print flag: no functions (0) / all functions (1) (numerical and fitted) are saved to unit luplot.

rdlow [1.D-02]

Lower limit of the random selection (used if iguess $=1$ ).

rdup $\quad[0.49 \mathrm{D} 0]$

Upper limit of the random selection (used if iguess=1)

\subsection{Input data for NUMCBAS}

Input data is read from standard input via a namelist /INPUT/. The size of the array parameters is indicated by the number of default values in brackets. The data type is indicated in the same way as for GTOBAS.

charge $\quad[0 . \mathrm{D} 0]$

Effective charge of the target (see section 2.1).

ecmax $[10 . \mathrm{D} 0]$

$E_{\text {up }}$, upper bound for eigenvalues in Rydberg.

hrx $\quad[0.01,0.02,0.02605,7 * 0 . \mathrm{D} 0]$

Vector of size nix containing the step length to be used in each subrange.

ibug $\quad[3 * 0]$

Vector containing switches for extra printed output: $\operatorname{ibug}(1)=1$ potential as a function of radial coordinate $r_{k} ; \operatorname{ibug}(2)=1$ final eigensolutions as function of $r_{k} ; \operatorname{ibug}(3)=1$ brief summary of data written to lunumb. 
irx $\quad\left[30,120,500,7^{*} 0\right]$

Vector of size nix defining the number of mesh points to end of each subrange (must be divisible by 2). The program checks that the last point in the last subrange is $\simeq \mathbf{r l i m}$. If it is not, a new $\operatorname{irx}(\mathbf{n i x})$ is calculated.

lunumb [13]

Logical unit for output of numerical functions.

lval $\quad[0]$

Angular momentum of the numerical functions to be calculated.

$\operatorname{nix} \quad[3]$

Number of subranges with different step. Maximum value is 10 .

rlim $\quad[10 . \mathrm{D} 0]$

$R_{\text {lim }}$, radius where the boundary condition is applied.

tiny $\quad[1 . \mathrm{D}-11]$

Convergence threshold for eigenvalues calculation.

title Character*80 variable containing title for output.

\section{Test Data}

Table 4 gives sample test data. Use of these data should give the exponents for $l=0$ presented in Tables 1 and 3 .

\section{Acknowledgements}

We thank Bernd Nestmann for helpful discussions on his work, and Peter Chocian and Andrew Richardson for work on the early stages of this project. This work was supported by the European Union by a Marie Curie Fellowship number CT-199900415 and by EPSRC. 


\section{References}

[1] P. W. Atkins, R. S. Friedman, Molecular quantum mechanics (Oxford University Press Inc., New York, Third edition, 1997).

[2] See Extensible Computational Chemistry Environment Basis Set Database, http://www.emsl.pnl.gov:2080/forms/basisform.html, mantained by the Environmental and Molecular Sciences Laboratory, Pacific Northwest Laboratory.

[3] K. Kaufmann, W. Baumeister and M. Jungen, J. Phys. B: At. Mol. Opt. Phys. 22 (1989) 2223.

[4] J. Tennyson and L. A. Morgan, Phil. Trans. R. Soc. Lond. A 357 (1999) 1161.

[5] B. M. Nestmann and S. Peyerimhoff, J. Phys. B: At. Mol. Opt. Phys. 23 (1990) L773.

[6] B. Sarpal, K. Pfingst, B. M. Nestmann and S. D. Peyerimhoff, J. Phys. B: At. Mol. Opt. Phys. 29 (1996) 857.

[7] T. M. Beyer, B. M. Nestmann, B. K. Sarpal and S. D. Peyerimhoff, J. Phys. B: At. Mol. Opt. Phys. 30 (1997) 3431.

[8] W. H. Klink and G. L. Payne, J. Comp. Phys. 21 (1976) 208.

[9] S. A. Salvini, Thesis, Queen's University of Belfast, 1983.

[10] L. A. Morgan, J. Tennyson and C. J. Gillan, Comput. Phys. Commun. 114 (1998) 120.

[11] R. de Vogelaere, J. Res. Nat. Bur. Standards B 54 (1955) 119.

[12] J. P. Coleman and J. Mohamed, Comput. Phys. Commun. 17 (1979) 283.

[13] M. J. D. Powell, Comput. J. 7, (1964) 155.

[14] D. L. Cooper, S. Wilson, J. Chem. Phys. 77 (1982) 5053.

[15] A. Faure and J. Tennyson, Mon. Not. R. Astron. Soc., (2001) in press.

[16] J. D. Gorfinkiel and J. Tennyson, in preparation.

[17] I. Rozum and J. Tennyson, in preparation.

[18] A. Faure and J.Tennyson, in preparation.

[19] A. E. Orel and K. C. Kulander, Phys. Rev. Lett. 71 (1993) 4315.

[20] W. H. Press, B. P. Flannery, S. A. Teukolsky and W. T. Vetterling, Numerical Recipes in Fortran (Cambridge University Press, 1995).

[21] J. Almlöf and P. R. Taylor in: Advanced Theories and Computational Approaches to electronic Structure of Molecules, C. E. Dykstra, ed. (Reidel, Dordrecht, 1984). 
[22] J. Almlöf and P. R. Taylor in: Modern Techniques in Computational Chemistry: MOTECC-91, E. Clementi, ed.(Escom, Leiden, 1991) Ch. 6A. 


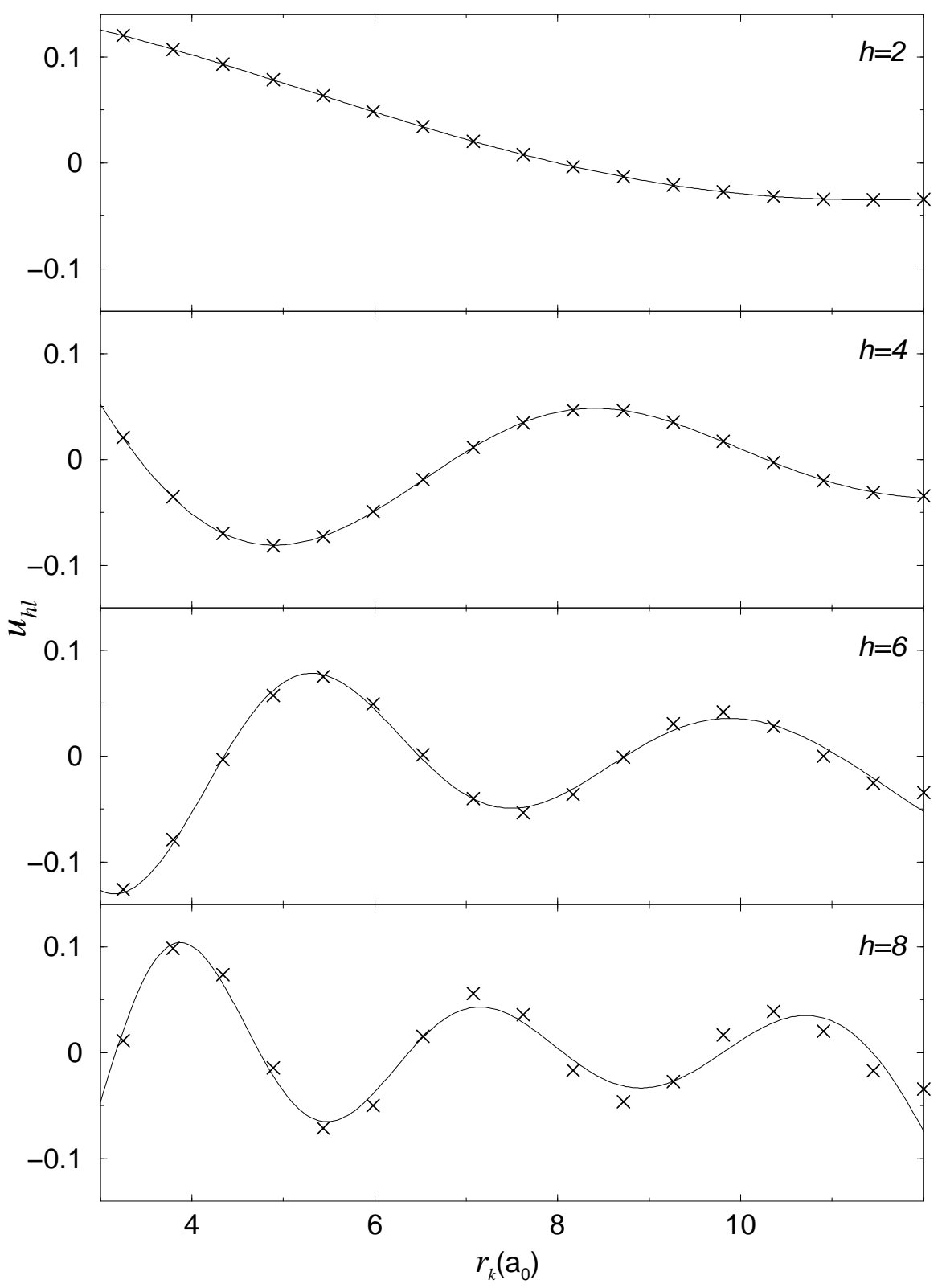

Fig. 1. Sample Bessel functions with $l=0$ (crosses) and their fit by a linear combination of 9 Gaussian functions (full curves) for $R_{\mathrm{lim}}=12 \mathrm{a}_{0}$ and $E_{\mathrm{up}}=4$ Ryd. $h$ indicates the specific function being plotted; the total number of nodes for each function is $h-1$. The exponents of the GTOs are those in the first column in Table 1. 




Fig. 2. Sample Coulomb functions with $l=0$ (crosses) and their fit by a linear combination of 12 Gaussian functions (full curves) for $R_{\text {lim }}=12 \mathrm{a}_{0}$ and $E_{\mathrm{up}}=5$ Ryd. $h$ and number of nodes as in Fig. 1. The exponents of the GTOs are those in the first column in Table 3. 


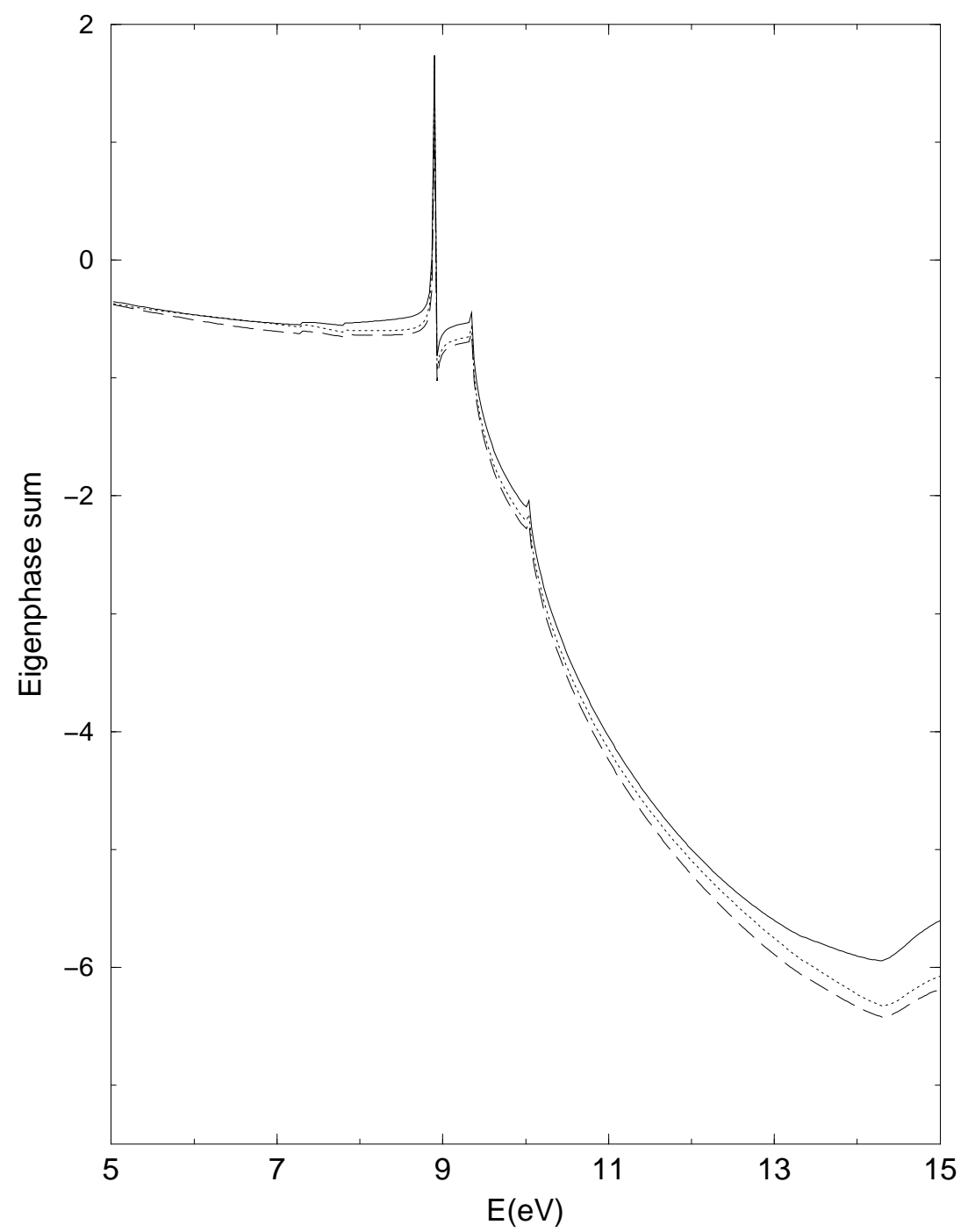

Fig. 3. Eigenphase sum of symmetry ${ }^{2} \mathrm{~A}_{1}$ for electron- $\mathrm{H}_{2} \mathrm{O}$ collisions. Full line: calculation for $R_{\text {mat }}=10 \mathrm{a}_{0}$ using basis set from Table 1; long-dashed line: $R_{\text {mat }}=10 \mathrm{a}_{0}$, using Sarpal et al.'s basis set; dotted line: calculation for $R_{m a t}=13 \mathrm{a}_{0}$ using basis set from Table 2. For a detailed description of these calculations, see [16]. 


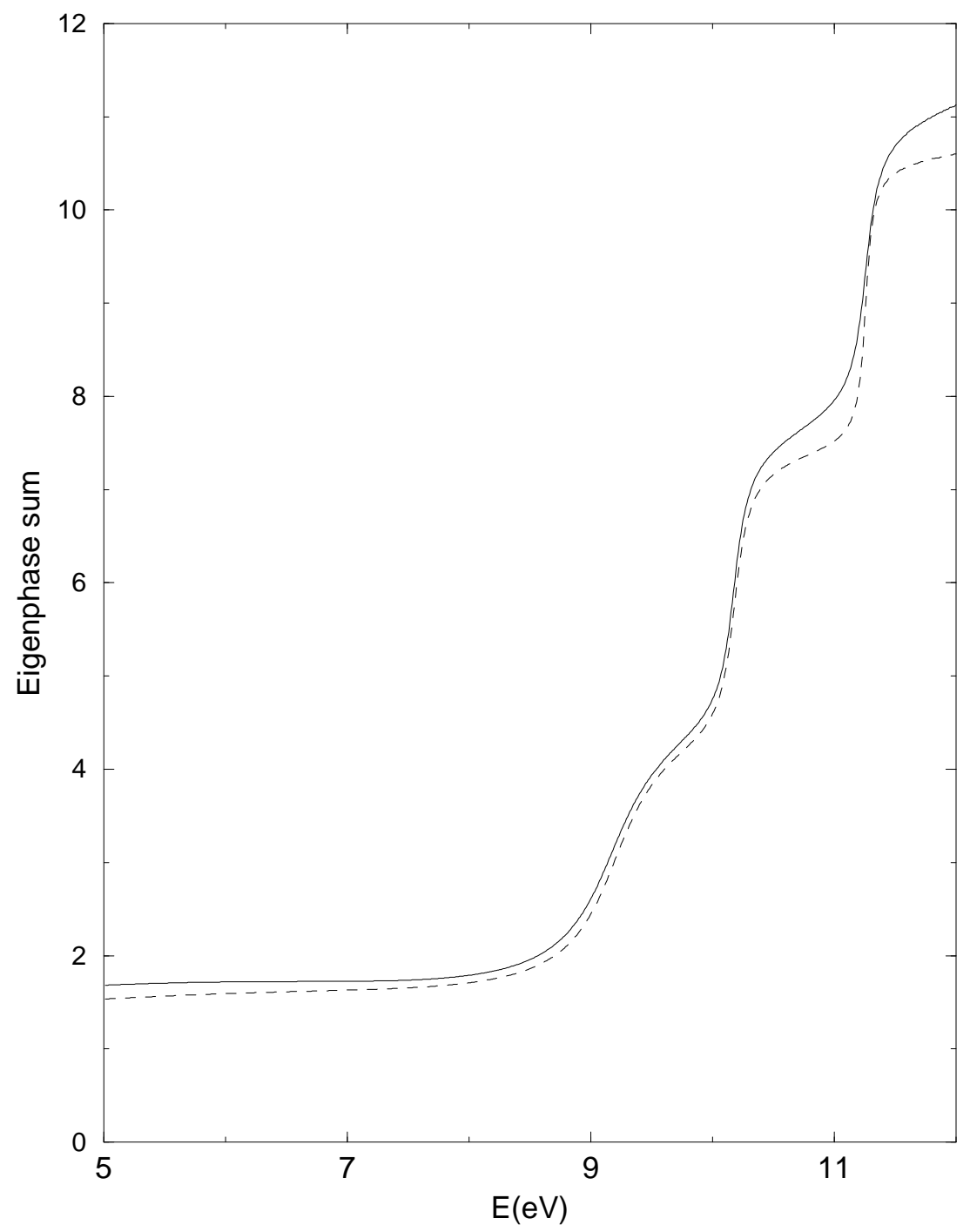

Fig. 4. Eigenphase sum of symmetry ${ }^{2} \mathrm{~A}_{1}$ for electron- $\mathrm{H}_{3}^{+}$collisions. Full line: calculation for $R_{m a t}=10 \mathrm{a}_{0}$ using basis set from Table 3; dashed line: $R_{m a t}=10 \mathrm{a}_{0}$, using Sarpal et al.'s basis set. For a detailed description of these calculations, see [18]. 
Table 1

Optimised GTO exponents for Bessel functions with $R_{\text {lim }}=12 a_{0}$ and $E_{\text {up }}=4$ Ryd. $N$ is the total number of Bessel functions for each $l$. Numbers in parentheses correspond to the basis set obtained by Sarpal et al. [6].

\begin{tabular}{|c|c|c|c|c|c|}
\hline & $\mathrm{s}(\mathrm{l}=0)$ & $\mathrm{p}(\mathrm{l}=1)$ & $\mathrm{d}(\mathrm{l}=2)$ & $\mathrm{f}(\mathrm{l}=3)$ & $\mathrm{g}(\mathrm{l}=4)$ \\
\hline $\mathrm{i} \backslash N$ & 8 & 7 & 7 & 6 & 6 \\
\hline 1 & $\begin{array}{c}.285726 \\
(.108108)\end{array}$ & $\begin{array}{c}.122916 \\
(.130267)\end{array}$ & $\begin{array}{c}.125926 \\
(.111252)\end{array}$ & $\begin{array}{c}.172844 \\
(.170320)\end{array}$ & $\begin{array}{c}.109047 \\
(.111363)\end{array}$ \\
\hline 2 & $\begin{array}{c}.192361 \\
(.095095)\end{array}$ & $\begin{array}{c}.090430 \\
(.102083)\end{array}$ & $\begin{array}{c}.094783 \\
(.089412)\end{array}$ & $\begin{array}{c}.125071 \\
(.130520)\end{array}$ & $\begin{array}{c}.083773 \\
(.089501)\end{array}$ \\
\hline 3 & $\begin{array}{c}.133124 \\
(.078078)\end{array}$ & $\begin{array}{c}.067262 \\
(.080605)\end{array}$ & $\begin{array}{c}.072171 \\
(.072361)\end{array}$ & $\begin{array}{c}.092858 \\
(.111586)\end{array}$ & $\begin{array}{c}.064982 \\
(.072433)\end{array}$ \\
\hline 4 & $\begin{array}{c}.092965 \\
(.063430)\end{array}$ & $\begin{array}{c}.049879 \\
(.063430)\end{array}$ & $\begin{array}{c}.054821 \\
(.058496)\end{array}$ & $\begin{array}{c}.069374 \\
(.089591)\end{array}$ & $\begin{array}{c}.050149 \\
(.058555)\end{array}$ \\
\hline 5 & $\begin{array}{c}.064850 \\
(.049698)\end{array}$ & $\begin{array}{c}.036582 \\
(.049698)\end{array}$ & $\begin{array}{c}.041223 \\
(.047005)\end{array}$ & $\begin{array}{c}.051579 \\
(.072505)\end{array}$ & $\begin{array}{c}.038123 \\
(.047052)\end{array}$ \\
\hline 6 & $\begin{array}{c}.044862 \\
(.038336)\end{array}$ & $\begin{array}{c}.026327 \\
(.038336)\end{array}$ & $\begin{array}{c}.030441 \\
(.037397)\end{array}$ & $\begin{array}{c}.037788 \\
(.058614)\end{array}$ & $\begin{array}{c}.028129 \\
(.035035)\end{array}$ \\
\hline 7 & $\begin{array}{c}.030582 \\
(.029180)\end{array}$ & $\begin{array}{c}.018347 \\
(.029180)\end{array}$ & $\begin{array}{c}.021766 \\
(.029225)\end{array}$ & $\begin{array}{c}.026876 \\
(.047099)\end{array}$ & \\
\hline 8 & $\begin{array}{c}.020403 \\
(.021923)\end{array}$ & & & & \\
\hline 9 & $\begin{array}{c}.013159 \\
(.013013)\end{array}$ & & & & \\
\hline
\end{tabular}


Table 2

Optimised GTO exponents for Bessel functions for $R_{\mathrm{lim}}=15 a_{0}, E_{\mathrm{up}}=5$ Ryd for $l=0,1,2, E_{\text {up }}=4$ Ryd for $l=3$ and $E_{\text {up }}=2.4$ Ryd fort $l=4$. $N$ is the total number of Bessel functions for each $l$.

\begin{tabular}{lccccc}
\hline & $\mathrm{s}(\mathrm{l}=0)$ & $\mathrm{p}(\mathrm{l}=1)$ & $\mathrm{d}(\mathrm{l}=2)$ & $\mathrm{f}(\mathrm{l}=3)$ & $\mathrm{g}(\mathrm{l}=4)$ \\
\hline $\mathrm{i} \backslash N$ & 11 & 10 & 10 & 8 & 6 \\
1 & .396150 & .200280 & .127610 & .092326 & .065228 \\
2 & .282361 & .151623 & .101449 & .073837 & .051981 \\
3 & .206334 & .117236 & .081731 & .059756 & .041790 \\
4 & .152887 & .091465 & .066052 & .048426 & .033499 \\
5 & .114144 & .071562 & .053288 & .039083 & .026564 \\
6 & .085455 & .055913 & .042273 & .031270 & .020594 \\
7 & .063902 & .043480 & .034058 & .024670 & \\
8 & .047570 & .033545 & .026815 & .018994 & \\
9 & .035148 & .025590 & .020778 & & \\
10 & .025705 & .019210 & .015690 & & \\
11 & .018550 & .014051 & & & \\
12 & .013150 & & & & \\
13 & .009071 & & & & \\
\hline & & & & & \\
\hline
\end{tabular}


Table 3

Optimised GTO exponents for Coulomb functions with $R_{\lim }=12 a_{0}, E_{\text {up }}=5$ Ryd for $l=0,1,2,3$ and $E_{\text {up }}=3$ Ryd for $l=4 . N$ is the total number of Coulomb functions for each $l$.

\begin{tabular}{lccccc}
\hline & $\mathrm{s}(\mathrm{l}=0)$ & $\mathrm{p}(\mathrm{l}=1)$ & $\mathrm{d}(\mathrm{l}=2)$ & $\mathrm{f}(\mathrm{l}=3)$ & $\mathrm{g}(\mathrm{l}=4)$ \\
\hline $\mathrm{i} \backslash N$ & 9 & 9 & 8 & 8 & 5 \\
1 & 40.9539 & 4.73096 & .186838 & .176575 & .096767 \\
2 & 3.19022 & .559667 & .136427 & .133689 & .071821 \\
3 & .460592 & .381904 & .101837 & .102897 & .053743 \\
4 & .292295 & .268948 & .076389 & .079368 & .039814 \\
5 & .194782 & .193043 & .057060 & .060885 & .028686 \\
6 & .132316 & .140037 & .042150 & .046164 & \\
7 & .090364 & .101932 & .030561 & .034350 & \\
8 & .061483 & .073983 & .021460 & .024755 & \\
9 & .041395 & .053241 & & & \\
10 & .027417 & .037780 & & & \\
11 & .017749 & .026258 & & & \\
12 & .011096 & .017649 & & & \\
\hline
\end{tabular}

Table 4

Tests data input for the numerical functions and corresponding fits represented in Figs. 1 and 2 .

\begin{tabular}{|c|c|c|}
\hline & BESSEL functions & COULOMB functions \\
\hline NUMCBA & $\begin{array}{l}\text { \&INPUT } \\
\text { title }=\text { 'Neutral target', } \\
\text { lval }=0 \\
\text { ecmax }=4.00 \mathrm{D} 0 \\
\text { rlim }=12.0 \\
\text { charge }=0.0 \mathrm{D} 0, /\end{array}$ & $\begin{array}{l}\& I N P U T \\
\text { title }=\text { 'Ionic target', } \\
\text { lval }=0, \\
\text { ecmax }=5.00 \mathrm{D} 0, \\
\text { rlim }=12.0 \mathrm{D} 0, \\
\text { charge }=1.0 \mathrm{D} 0, /\end{array}$ \\
\hline GTOBAS & $\begin{array}{l}\& \mathrm{FIT} \\
\text { expo }=6.0,3.0,0.7,0.3,0.1,0.07,0.05,0.02,0.01, \\
\text { iguess }=0, \\
\text { nplot }=1, \\
\text { iprint }=0, /\end{array}$ & $\begin{array}{l}\& \text { FIT } \\
\text { noexp }=12 \\
\text { iguess }=2 \\
\text { nplot }=1 \\
\text { iprint }=0, /\end{array}$ \\
\hline
\end{tabular}

